\title{
Comprehensive analysis of the Ppatg3 mutant reveals that autophagy plays important roles in gametophore senescence in Physcomitrella patens
}

Zexi Chen ${ }^{1,2+}$, Wenbo Wang ${ }^{1,2,3+}$, Xiaojun Pu ${ }^{1}$, Xiumei Dong ${ }^{1}$, Bei Gao ${ }^{4}$, Ping Li ${ }^{1}$, Yanxia Jia ${ }^{5}$, Aizhong Liu ${ }^{1,6}$ and Li Liu ${ }^{1,7^{*}}$ (D)

\begin{abstract}
Background: Autophagy is an evolutionarily conserved system for the degradation of intracellular components in eukaryotic organisms. Autophagy plays essential roles in preventing premature senescence and extending the longevity of vascular plants. However, the mechanisms and physiological roles of autophagy in preventing senescence in basal land plants are still obscure.

Results: Here, we investigated the functional roles of the autophagy-related gene PpATG3 from Physcomitrella patens and demonstrated that its deletion prevents autophagy. In addition, Ppatg3 mutant showed premature gametophore senescence and reduced protonema formation compared to wild-type (WT) plants under normal growth conditions. The abundance of nitrogen ( $\mathrm{N}$ ) but not carbon (C) differed significantly between Ppatg3 mutant and WT plants, as did relative fatty acid levels. In vivo protein localization indicated that PpATG3 localizes to the cytoplasm, and in vitro Y2H assays confirmed that PpATG3 interacts with PpATG7 and PpATG12. Plastoglobuli (PGs) accumulated in Ppatg3, indicating that the process that degrades damaged chloroplasts in senescent gametophore cells was impaired in this mutant. RNA-Seq uncovered a detailed, comprehensive set of regulatory pathways that were affected by the autophagy mutation.
\end{abstract}

(Continued on next page)

\footnotetext{
* Correspondence: liulia@mail.kib.ac.cn

${ }^{\dagger}$ Zexi Chen and Wenbo Wang contributed equally to this work.

'Department of Economic Plants and Biotechnology, Yunnan Key Laboratory

for Wild Plant Resources, Kunming Institute of Botany, Chinese Academy of Sciences, Kunming 650204, China

${ }^{7}$ State Key Laboratory of Biocatalysis and Enzyme Engineering, Hubei Collaborative Innovation Center for Green Transformation of Bio-Resources, Hubei Key Laboratory of Industrial Biotechnology, School of Life Sciences, Hubei University, Wuhan 430062, China

Full list of author information is available at the end of the article
}

(C) The Author(s). 2020 Open Access This article is licensed under a Creative Commons Attribution 4.0 International License, which permits use, sharing, adaptation, distribution and reproduction in any medium or format, as long as you give appropriate credit to the original author(s) and the source, provide a link to the Creative Commons licence, and indicate if changes were made. The images or other third party material in this article are included in the article's Creative Commons licence, unless indicated otherwise in a credit line to the material. If material is not included in the article's Creative Commons licence and your intended use is not permitted by statutory regulation or exceeds the permitted use, you will need to obtain permission directly from the copyright holder. To view a copy of this licence, visit http://creativecommons.org/licenses/by/4.0/ The Creative Commons Public Domain Dedication waiver (http://creativecommons.org/publicdomain/zero/1.0/) applies to the data made available in this article, unless otherwise stated in a credit line to the data. 


\begin{abstract}
(Continued from previous page)
Conclusions: The autophagy-related gene PpATG3 is essential for autophagosome formation in P. patens. Our findings provide evidence that autophagy functions in $\mathrm{N}$ utilization, fatty acid metabolism and damaged chloroplast degradation under non-stress conditions. We identified differentially expressed genes in Ppatg3 involved in numerous biosynthetic and metabolic pathways, such as chlorophyll biosynthesis, lipid metabolism, reactive oxygen species removal and the recycling of unnecessary proteins that might have led to the premature senescence of this mutant due to defective autophagy. Our study provides new insights into the role of autophagy in preventing senescence to increase longevity in basal land plants.
\end{abstract}

Keywords: Autophagy defect, ATG, C/N ratio, Fatty acid, Chloroplast plastoglobuli, Premature senescence, Moss

\section{Background}

Autophagy is an evolutionarily conserved, ubiquitous process in eukaryotic cells that degrades damaged or toxic intracellular components for recycling to maintain essential cellular functions and life activities [1-3]. In plants, autophagy contributes to nutrient use efficiency and energy metabolism and is upregulated during senescence to promote cellular homeostasis and longevity [46]. Two types of autophagy pathways have been identified in plants: macroautophagy and microautophagy [7]. Macroautophagy, which had been extensively studied, is regulated by AuTophaGy (ATG) genes, whose expression results in the formation of a double-membrane organelle known as the autophagosome [2]. Bulk cytosolic components, including organelle fragments and macromolecules, are then transferred into the vacuole via fusion with the autophagosome and are subsequently degraded by lytic enzymes within the vacuole. We use the term 'autophagy' hereafter to refer specifically to macroautophagy. To date, at least 30 ATG proteins had been identified in yeast (Saccharomyces cerevisiae), which can be divided into several functional classes: a) the ATG1ATG13 kinase complex; b) ATG9 and ATG9-associated proteins; c) the phosphatidylinositol 3-kinase complex; and d) two ubiquitin-like conjugation systems mediated by ATG8 or ATG12 [8]. Most of these proteins have homologs in plants. Autophagy plays multiple physiological roles in plants, functioning in processes such as biotic and abiotic stress responses $[9,10]$, anther development [11], leaf starch degradation [12], lipid/fatty acid homeostasis and turnover [11, 13-15], damaged chloroplast degradation $[16,17]$, soluble/aggregated protein degradation [18] and senescence [2, 19]. ATG3 is an E2-like enzyme involved in the ATG8 and phosphatidylethanolamine (PE) conjugation system during autophagosome formation [20]. Based on the crystal structure of S. cerevisiae ATG3, cysteine 234 (Cys-234) is the active residue that is important for the lipidization reaction of ATG8$\mathrm{PE}$ conjugation [21]. Autophagosome formation is defective in ATG3 mutant in yeast [22] and Toxoplasma [23], and autophagic activity was enhanced by overexpressing ATG3 in tobacco [24].
Autophagy is a fundamental factor in cell longevity and senescence in eukaryotes, especially plants $[2,4]$. The recycling and remobilization of nutrients, including carbon $(\mathrm{C})$ and nitrogen $(\mathrm{N})$, are crucial for plant survival and adaptation, especially under nutrient-limiting conditions [25]. Recent reports in Arabidopsis thaliana (Arabidopsis) revealed that autophagy is important for N-remobilization efficiency [26-28] and controls the C/ $\mathrm{N}$ ratio [29]. However, to date, most studies in Arabidopsis on the roles of autophagy in nitrogen utilization and senescence were conducted under nutrient starvation or abiotic stress conditions, and few studies have focused on these processes under normal growth conditions. Moreover, recent studies suggested that autophagy plays important roles in lipid/fatty acid metabolism [11], composition [13] and turnover [14] in several vascular plants, although whether autophagy affects fatty acids in basal land plants is unknown. Even though autophagy is known to be essential for $\mathrm{C} / \mathrm{N}$ status and lipid/fatty acid metabolism in plants, the details of the autophagy regulatory machinery are mostly unknown.

Physcomitrella patens, a basal land plant commonly used for developmental biology research, had been used to study autophagy during senescence in the dark [30] and during gamete differentiation [31]. However, to date, only two autophagy genes, ATG5 and ATG7, have been identified and studied in $P$. patens. Further elucidation of the regulatory pathway of ATGs in moss would increase our understanding of the roles of autophagy in plant development. In the current study, we analyzed ATG3 knockout $P$. patens lines under normal growth conditions. The gametophores of the mutant displayed early-senescence symptoms, including yellowing, impaired photosynthesis, reduced chlorophyll levels, the accumulation of chloroplast plastoglobuli (PGs) and differential expression of senescence-associated genes (SAGs) under normal growth conditions. Analysis of whole-plant $\mathrm{C} / \mathrm{N}$ ratios and fatty acid contents revealed that autophagy plays essential roles in N-utilization efficiency and fatty acid metabolism in $P$. patens gametophores. In addition, we performed comprehensive RNASeq analysis to provide insight into the role of autophagy 
in gametophore senescence in $P$. patens. Our study provides evidence for the role of autophagy in $\mathrm{N}$ utilization, fatty acid/lipid metabolism, damaged chloroplast degradation, reactive oxygen species (ROS) removal and recycling of unnecessary proteins under non-stress conditions to prevent senescence and enhance cell longevity in the basal land plant $P$. patens.

\section{Results}

\section{Identification of ATG3 from $P$. patens}

The 924-bp PpATG3 coding sequence contains 9 exons and is almost the same size as Arabidopsis ATG3 (AT5G61500, $942 \mathrm{bp}$, with 9 exons). Protein sequence alignment revealed both conservation and divergence of the three primary functional domains of ATG3 proteins in $P$. patens vs. Klebsormidium nitens, Mesotaenium endlicherianum, Anthoceros angustus, Marchantia polymorpha, Brachypodium distachyon, A. thaliana, S. cerevisiae, Mus musculus and Homo sapiens (Additional file 1A). Two of these domains (Autophagy_act_C and Autophagy_C) showed high levels of conservation, while the third (Autophagy_N) was weakly conserved. Notably, the Autophagy_C domain was missing in the subaerial green alga Mesotaenium endlicherianum (MeATG3). In addition, the three domains of ATG3 were more conserved within plants vs. animals. However, the key, functionally necessary Cys-234 residue was detected in the ATG3s of all species. Nineteen amino acids were highly conserved among plant species but differed from those of yeast and human/mouse.

We predicted the secondary structures of the ATG3s based on the crystal structure of ScATG3 (Additional file 1A). The Autophagy_N, Autophagy_act_C and Autophagy_C domains comprise three alpha helices $(\alpha 1$, $\alpha 2, \alpha 3)$ and two beta sheets $(\beta 1, \beta 2)$, two alpha helices $(\alpha 4, \alpha 5)$ and three beta sheets $(\beta 4, \beta 5, \beta 6)$, and one alpha helix $(\alpha 7)$, respectively. $\beta 3$ is partially contained in the Autophagy_act_C domain, and $\alpha 6$ is positioned between the Autophagy_act_C and Autophagy_C domains. Eight motifs $(1,2,3,4,5,6,8$ and 10) are present ATG3 proteins from both plants and animals, while two motifs (7 and 9) are present only in plants (Additional file 1B). Sequence alignment and motif analysis pointed to the divergence of ATG3s between plants and animals. Phylogenetic analysis also showed that the ATG3 genes were clustered into two different clades (Additional file $1 C)$. These results indicate that these genes have undergone early divergence and independent evolution between the plant and animal lineages. In addition, the conserved characteristics of ATG3 between land plants and subaerial green algae suggest that the functional divergence of these genes occurred prior to land plant terrestrialization.
Tissue-specific expression profiles and subcellular localization of PpATG3

To assess the expression patterns of PpATG3 in different tissues, we retrieved the corresponding microarray data from the transcriptome of $P$. patens [32]. PpATG3 was expressed at high levels throughout the $P$. patens life cycle, with transcript abundance (robust multi-array average) values $>5000$ (Fig. 1a). To determine the subcellular localization of PpATG3 in P. patens, we fused the full-length coding sequence of PpATG3 with that of enhanced green fluorescent protein (eGFP) in-frame under the control of the constitutive CaMV35S promoter (p35S:PpATG3-eGFP) and transiently transformed $P$. patens protoplasts with this construct (Fig. 1b). We used the empty vector (EV) as a control (Fig. 1b). Confocal microscopy revealed that the fluorescent signal of the PpATG3-eGFP fusion proteins was evenly distributed in the cytoplasm of the protoplasts, whereas the EV control did not generate a signal.

\section{PpATG3 knockout disrupts gametophore senescence and protonema formation}

To further explore the role of PpATG3, we generated Ppatg3 knockout transgenic plants by disrupting exons 4 and 5 through homologous recombination (HR) (Fig. 1c). This yielded three knockout lines (ko\#22, ko\#31 and ko\#50) of PpATG3, whose identities were confirmed by PCR. We isolated genomic DNA and total RNA from these plants to verify the genomic insertion of the nptII cassette and loss of PpATG3 transcripts due to HR events at its $5^{\prime}$ and $3^{\prime}$ flanks (Fig. 1c), respectively. PpATG3 had been successfully disrupted at the genomic locus via the insertion of a 2078-bp nptII cassette into both arms of the target by HR. To investigate whether PpATG3 functions in gametophore development in $P$. patens, we examined 7- to 56-day-old wild-type (WT) and Ppatg3 knockout plants under normal growth conditions. There was a significant difference between Ppatg3 knockout and WT plants, with the mutant showing an increasingly premature-senescence phenotype over time (Fig. 2a). The chlorophyll fluorescence of the Ppatg3 mutant became weaker than that of WT plants (Fig. 2a). This premature senescence was most notable in 56-day-old plants, as the stem sections and basal leaves of leafy gametophores in the Ppatg3 knockout plants turned yellow (Fig. 2b). In addition, in 56-old-day plants, there were far fewer newly formed protonemata in Ppatg3 knockout plants compared to WT plants (Fig. 2b, red circles).

The photosynthetic yield ( $\mathrm{Fv} / \mathrm{Fm})$ values also differed in 7-day-old Ppatg3 knockout vs. WT plants, and subsequently the mutant showed seriously decreased fluorescence compared to WT plants (Fig. 2c). This finding is supported by the reduced chlorophyll biosynthesis in 


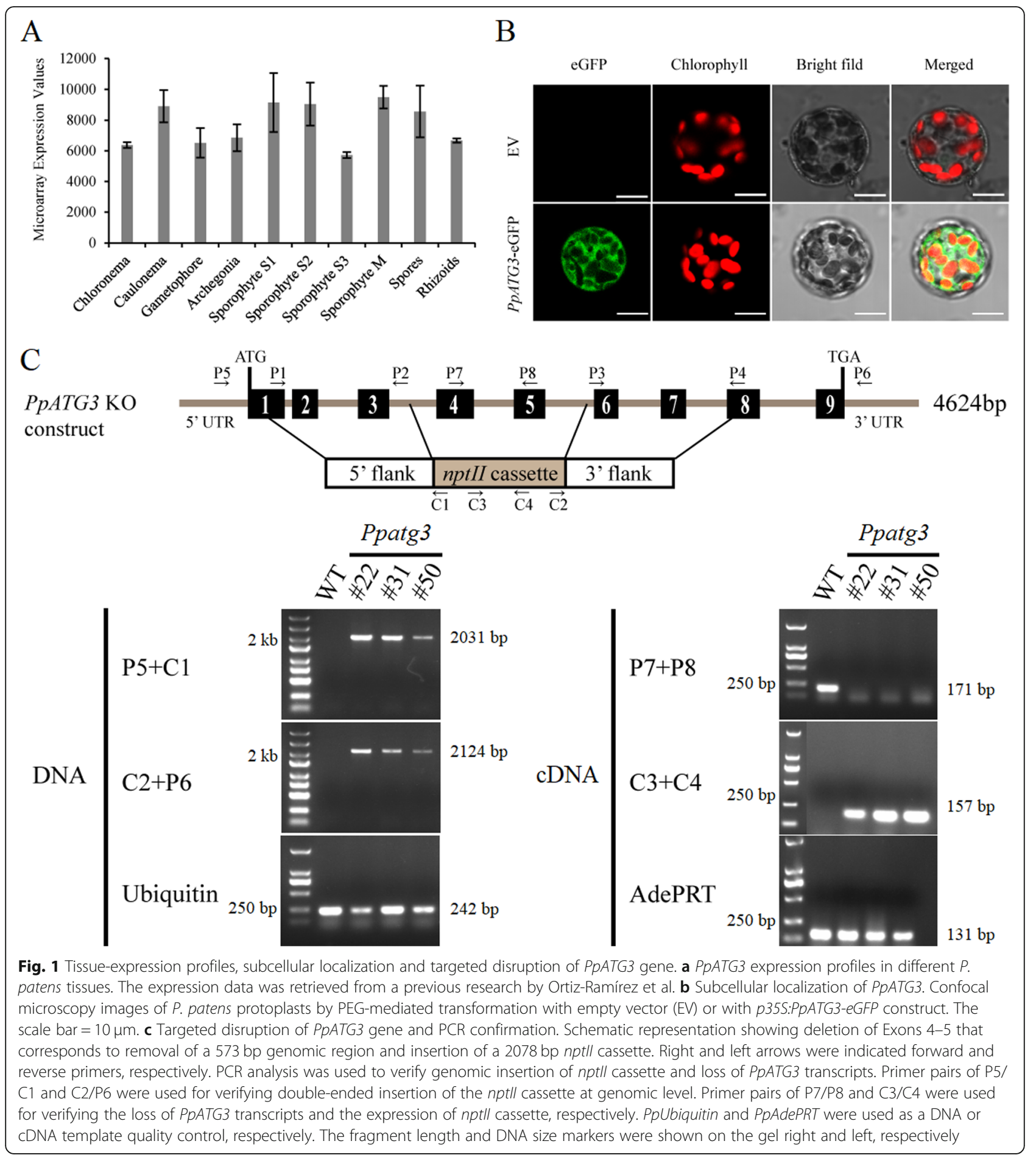

Ppatg3 knockout plants: the chlorophyll a, chlorophyll b and total chlorophyll contents were significantly lower in both 14- and 28-day-old Ppatg3 mutant vs. WT plants (Fig. 2d). However, the chlorophyll contents were also slightly lower in 28-day-old WT plants than in 14-dayold WT plants, likely because more protonemata were present in younger plants. These results indicate that the
Ppatg3 knockout plants underwent a greater reduction in chlorophyll content than the WT, resulting in an early-senescence phenotype.

PpATG3 dysfunction affects cell development in P. patens To explore how PpATG3 regulates plant senescence, we examined the leafy gametophores cells of WT and Ppatg3 

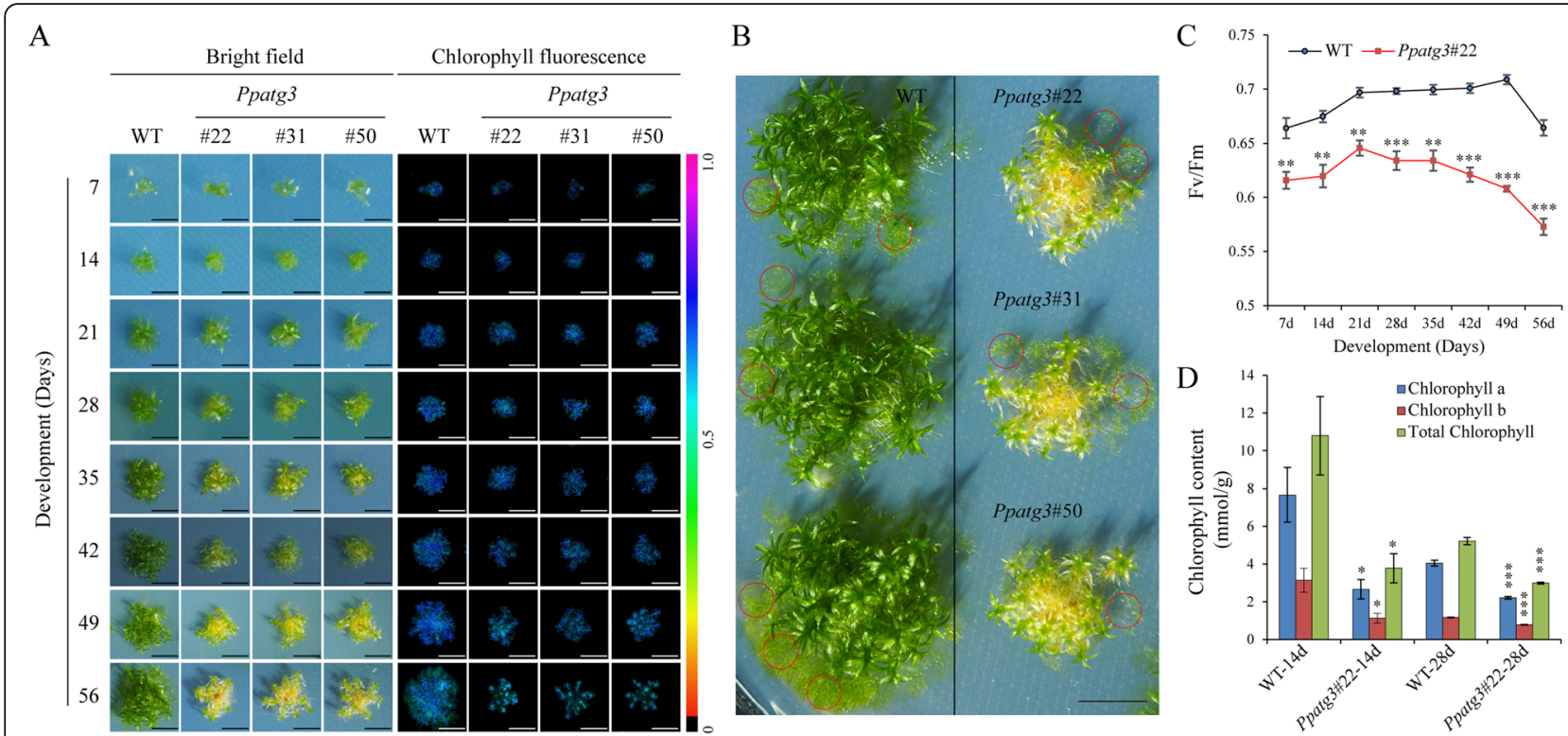

Fig. 2 PpATG3 affects growth and photosynthetic regulation in P. patens. a WT and Ppatg3 knockout plants were observed after growing 7 to 56 days at normal growth conditions. The scale bar $=4 \mathrm{~mm}$. $\mathbf{b}$ PpATG3 affects the formation of new protonemata. The 56-day-old plants were used for analysis and the red circles were indicated newly formed protonemata. The scale bar $=4 \mathrm{~mm}$. $\mathbf{c}$ Fv/Fm values of WT and Ppatg3 plants. $\mathbf{d}$ Chlorophyll decreased in the Ppatg3 knockout plants. Three biological replicates were analyzed and error bars show the mean value \pm SD. The asterisks indicate a significant change between the Ppatg3 and WT plants at $\left(^{*}\right) p<0.05$, $\left(^{* *}\right) p<0.01$, and $\left(^{* *}\right) p<0.001$

plants grown under normal conditions in detail. The cells of the Ppatg3 mutant appeared hollow and were turning yellow, whereas those of WT plants remained full and green (Fig. 3a and b). To validate that the deletion of PpATG3 prevents autophagosome formation in $P$. patens, we treated 28 day-old WT and Ppatg3 knockout plants with $100 \mathrm{mM}$ $\mathrm{NaCl}$ for $1 \mathrm{~h}$ and observed them by transmission electron microscopy (TEM). Autophagosomes containing cellular cargos formed in WT plants (Fig. 3c), whereas the bulk cytosolic components accumulated in the mutant due to PpATG3 knockout (Fig. 3d). These results indicate that autophagy was disrupted in the Ppatg3 mutant.

To further explore the effect of PpATG3 on senescence in moss, we examined chloroplasts in WT and Ppatg3 cells. We observed a higher density of cellular substances in leafy gametophore cell from the Ppatg3 mutant compared to WT (Fig. 3e and f). Moreover, in the mutant, these cells accumulated an unusually high density of chloroplast PGs; these lipoprotein particles play important roles in various metabolic processes such as photosynthetic regulation, thylakoid lipid remobilization and senescence [33]. The higher density of chloroplast PGs in Ppatg3 leafy gametophore cells suggests that PGs accumulation might be related to the reduced chlorophyll levels in the autophagy mutant.

\section{Changes in $\mathrm{C} / \mathrm{N}$ ratios and fatty acid contents}

The $\mathrm{C} / \mathrm{N}$ ratio is reduced in Arabidopsis autophagy mutant [29], and lipid metabolism is impaired in rice
OsATG7 knockout mutant [11]. Based on the hypothesis that changes in $\mathrm{C} / \mathrm{N}$ ratios and fatty acid contents caused the early-senescence phenotype seen in Ppatg3 knockout plants, we measured the $\mathrm{C} / \mathrm{N}$ ratios of WT and Ppatg3 plants at three time points: 14, 28 and 56 days (Fig. $4 \mathrm{a}-\mathrm{C}$ ). At 14 days, we did not detect any significant differences in $\mathrm{C}$ or $\mathrm{N}$ concentrations or $\mathrm{C} / \mathrm{N}$ ratios between Ppatg3 knockout and WT plants. At 28 and 56 days, however, Ppatg3 plants showed notably lower $\mathrm{C} / \mathrm{N}$ ratios than the WT due to higher $\mathrm{N}$ contents (N\%). Overall, the N\% rates gradually decreased over the three time points in WT plants, whereas they remained constant in Ppatg3 knockout plants. These results suggest that $\mathrm{N}$ utilization was completely defective in the Ppatg3 mutant. Notably, the $\mathrm{C}$ contents $(\mathrm{C} \%)$ did not significantly differ between Ppatg3 knockout and WT plants.

Beike et al. [34] detected high fatty acid (\%) contents in the gametophores of wild-type P. patens. Here, we analyzed the contents of six fatty acids in $P$. patens: palmitic acid (16:0), stearic acid (18:0), oleic acid (18:1), linoleic acid (18:2), arachidic acid (20:0) and arachidonic acid (20:4). We chose two time points: 28 and 56 days (Fig. $4 \mathrm{~d}-\mathrm{e}$ ). At 28 days, three fatty acids (palmitic acid, oleic acid and arachidic acid) showed significantly higher relative abundance (\%) and two (linoleic acid and arachidonic acid) showed significantly lower relative abundance in the Ppatg3 mutant compared to the WT. Similarly, at 56 days, three fatty acids (palmitic acid, 

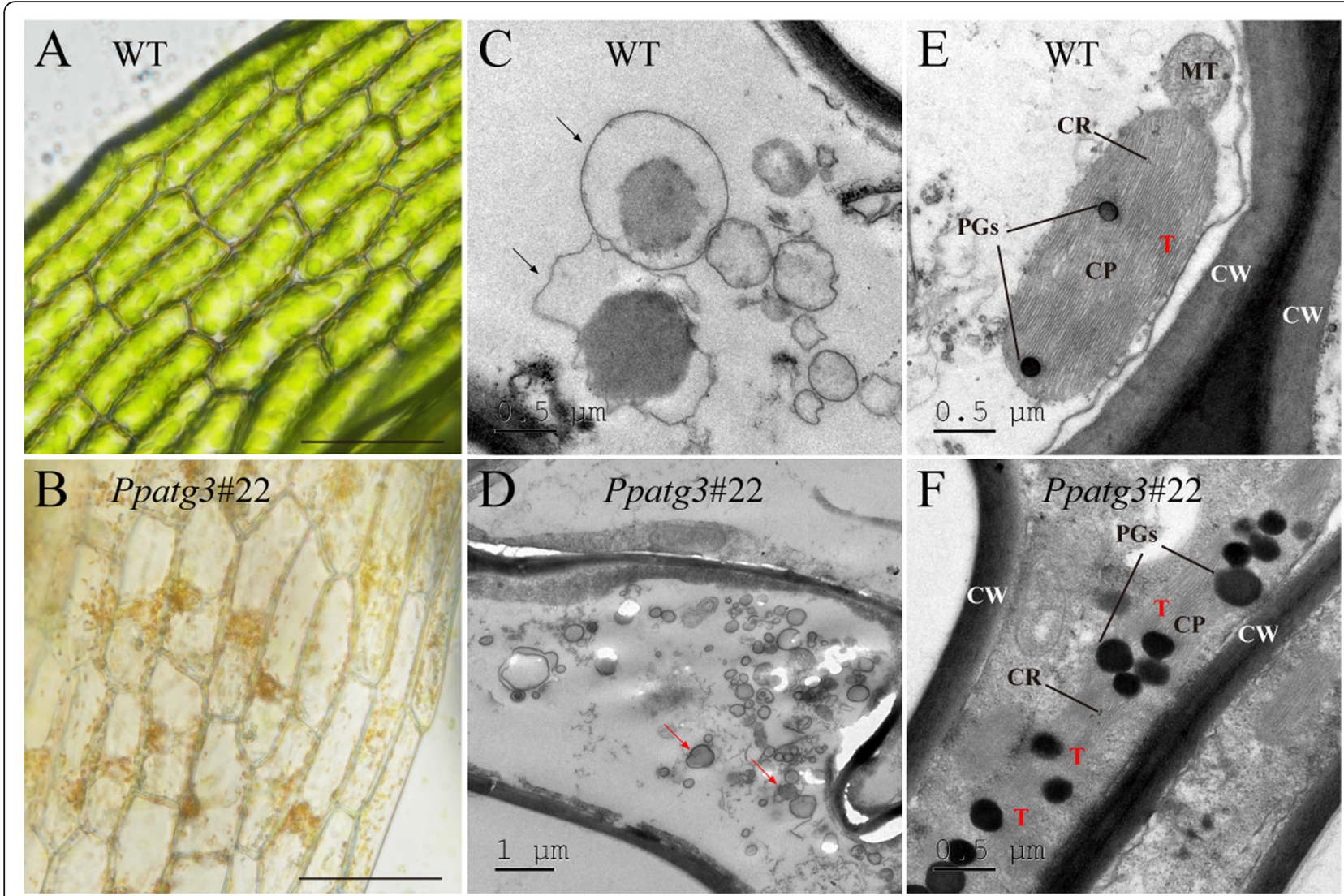

Fig. 3 PpATG3 affects the cell development in P. patens. $\mathbf{a}$ and $\mathbf{b}$ Leafy gametophore cells were observed by light microscopy. The scale bar $=0.3$ mm. $\mathbf{c}$ and $\mathbf{d}$ Detection of autophagosome in the gametophore cells of WT and Ppatg3 knockout plants by TEM. The 28-day-old plants after treatment for $1 \mathrm{~h}$ of $100 \mathrm{mM} \mathrm{NaCl}$ were used for analysis. The black arrows were indicated the formation of autophagosomes in WT and the red arrows were indicated the bulk cytosolic components accumulated in Ppatg3 mutants due to autophagy defect. e and $\mathbf{f}$ PpATG3 dysfunction causes the accumulation of chloroplast plastoglobuli. The 28-day-old plants at normal growth conditions were used for analysis. CP, chloroplast; PGs, plastoglobuli; T, thylakoid; CR, chloroplast ribosome; MT, mitochondrion; CW, cell wall

stearic acid and arachidic acid) showed significantly higher and three (oleic acid, linoleic acid and arachidonic acid) showed significantly lower relative abundance in Ppatg3 vs. the WT. By contrast, the relative stearic acid contents did not significantly differ between Ppatg3 and WT plants at 28 days. Overall, the fatty acid profiles markedly differed between Ppatg3 knockout and WT plants.

To further investigate the relationship between $\mathrm{C} / \mathrm{N}$ ratio and fatty acid contents in the autophagy-defective mutant, we performed a fatty acid supplementation experiment. Because the linoleic acid and arachidonic acid contents were significantly reduced in the Ppatg3 mutant (Fig. 4d-e), we hypothesized that these two fatty acids function in $\mathrm{C} / \mathrm{N}$ status in $P$. patens. Indeed, supplementing WT plants with linoleic and arachidonic acids, either singly or together, altered the $\mathrm{C} / \mathrm{N}$ status and decreased the $\mathrm{C} / \mathrm{N}$ ratio compared to the control (Additional file 2A-C). By contrast, supplementing Ppatg3 plants with linoleic acid or arachidonic acid alone did not improve $\mathrm{N}$ utilization, and supplementation with both linoleic acid and arachidonic acid reduced the $\mathrm{N}$ contents, resulting in a $\mathrm{C} / \mathrm{N}$ ratio similar to that of WT plants (Additional file $2 \mathrm{~A}-\mathrm{C}$ ). However, the premature gametophore senescence phenotype of the mutant was not rescued by fatty acid supplementation (Additional file 2D).

\section{RNA-Seq to identify differentially expressed genes in Ppatg3}

To examine whether the loss of ATG3 affects the gene expression profile of $P$. patens, we analyzed the global gene expression pattern of the Ppatg3 mutant compared to the WT control using the BGISEQ-500 platform. Transcripts with FPKM $\geq 1$ were subjected to further analysis. PCA revealed highly significant transcriptional differences between Ppatg3 and WT plants (Additional file 3 A). In total, 23,219/16,564 expressed transcripts/ genes were detected from all samples, including 23,139/ 16,503 transcripts/genes expressed in both Ppatg3 and 


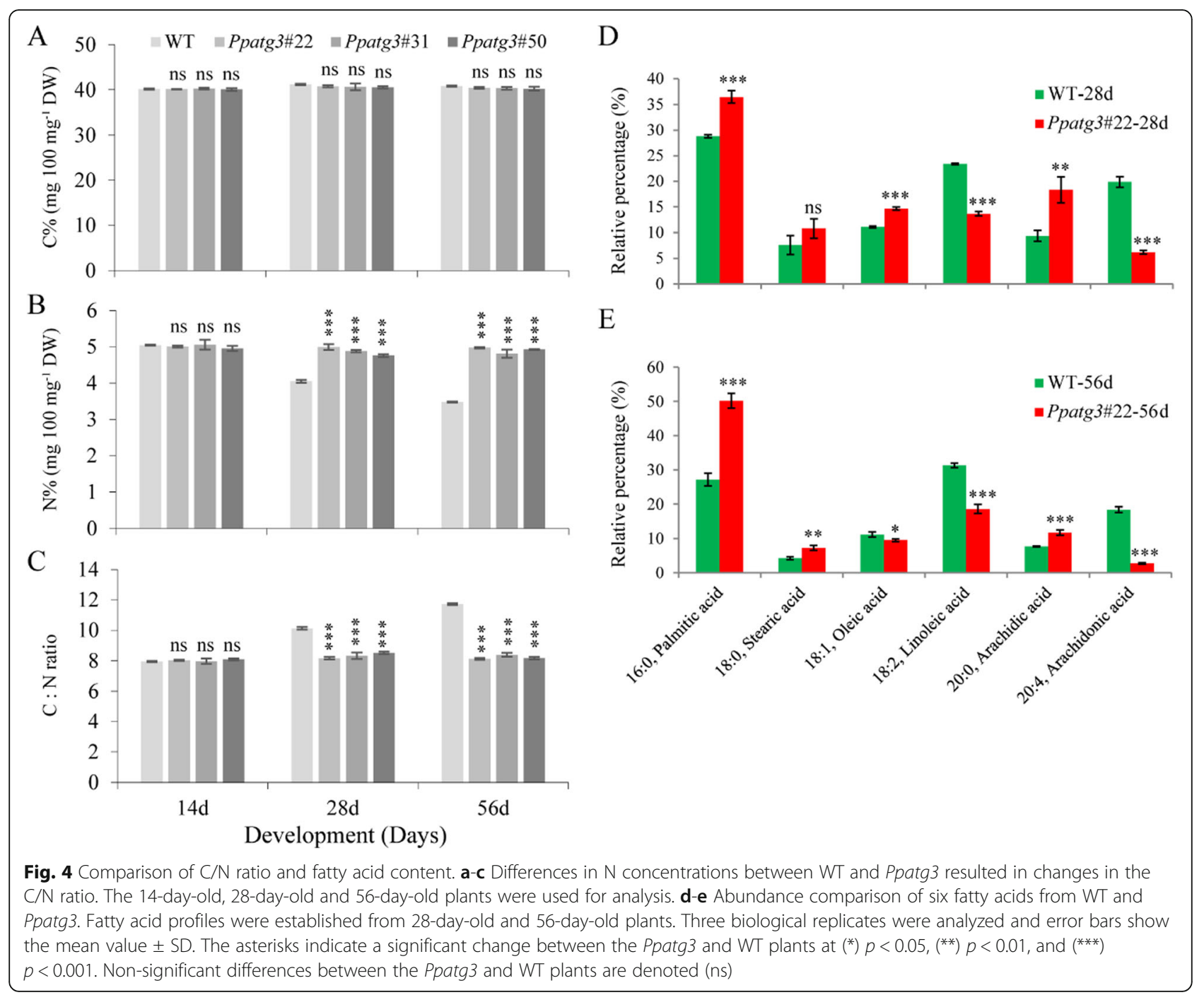

WT plants, 45/38 unique transcripts/genes in Ppatg3 and 35/23 unique transcripts/genes in WT (Additional file $3 \mathrm{~B}$ and Additional file 4). Using the criteria of $p$ value $\leq 0.001$ and expression fold change $>2$ to identify differentially expressed transcripts/genes (DETs/DEGs), a comparison of Ppatg3 and WT revealed a total of 3080/2634 DETs/DEGs. Of these, 1845/1621 DETs/ DEGs and 1235/1013 DETs/DEGs were upregulated and downregulated, respectively, in Ppatg3 vs. the WT (Additional file 5).

We then identified the top 20 enriched KEGG pathways of the up- and downregulated DETs/DEGs at $Q$ value $\leq 0.05$ (Additional file $3 \mathrm{D}-\mathrm{E}$ and Additional file 6). Among both up- and downregulated DETs/DEGs, the enriched pathways were all biosynthetic and metabolic pathways, which were roughly divided into five major functional classes: carbohydrate metabolism, energy metabolism, amino acid metabolism, cofactor and vitamin metabolism, and global pathways. Notably, the nitrogen metabolism pathway was significantly enriched (Additional file $3 \mathrm{D}$ ), which might be related to the altered $\mathrm{N}$ contents of the Ppatg3 mutant.

In Arabidopsis, the differential expression of senescence-associated genes (SAGs) and weakened photosynthetic capacity are associated with plant senescence [35]. Notably, numerous genes related to chlorophyll biosynthesis and photosystems were downregulated in Ppatg3 vs. the WT (Additional file 7). In addition, half of the SAGs (11 of 22) were significantly upregulated in the Ppatg3 mutant compared to the WT (Additional file 7). These results provide evidence for the accelerated senescence process in the Ppatg3 mutant.

\section{The transcription of nitrogen and fatty acid/lipid} metabolism-related genes is altered in the Ppatg 3 mutant To further investigate the reason for the dysfunctional $\mathrm{N}$ and fatty acid metabolism in Ppatg3, we compared the 
differences in transcript levels of genes related to nitrogen and fatty acid/lipid metabolism. Ten of the 11 genes were significantly upregulated, including genes related to glutamine synthetase (GLN), glutamate synthase (GLS), nitrate reductase (NR) and glutamate dehydrogenase (GDH) (Fig. 5a and Additional file 8). These results indicate that the nitrogen metabolism pathway was defective in Ppatg3, resulting in the differential expression of genes involved in nitrogen metabolism. This phenomenon might be due to feedback regulation of nitrogen-related DEGs caused by a N-utilization deficiency in the Ppatg3 mutant. However, the upregulated expression of these genes did not restore the $\mathrm{N}$ utilization efficiency, suggesting that the regulation mechanism of autophagy for $\mathrm{N}$ utilization was more complicated.

Furthermore, 12 genes related to fatty acid biosynthesis and metabolism were significantly differentially expressed in the mutant, including 7 upregulated and 5 downregulated genes (Fig. 5b and Additional file 8). One upregulated gene, the lipoxygenase homologous gene (LOX5; Pp3c1_29300), might be involved in linoleic acid metabolism; its higher expression level is consistent with the reduced linoleic acid contents in Ppatg3. However, another lipoxygenase homologous gene (LOX3; Pp3c15 13040), which might be involved in the arachidonic acid metabolism, was downregulated in the mutant: its lower expression level might not be related to the reduced arachidonic acid contents in Ppatg3. Moreover, 19 of the 30 genes related to lipid metabolism were significantly upregulated in Ppatg3, including genes involved in glycerolipid, glycerophospholipid and sphingolipid metabolism (Fig. 5c and Additional file 8).

\section{Dysfunctional autophagy leads to the differential transcription of protein metabolism, endocytosis and ROS-related genes}

Twenty-five out of 31 ubiquitin-related genes were significantly upregulated in the Ppatg3 mutant vs. the WT (Fig. 6a and Additional file 8). These highly expressed genes encode proteins including ubiquitin proteins or regulators, ubiquitin-activating enzymes (E1), ubiquitinconjugating enzymes (E2) and ubiquitin ligases (E3). Moreover, the transcription of genes in the $26 \mathrm{~S}$ proteasome system was activated by the upregulation of a subset of regulatory genes in the mutant (Fig. 6b and
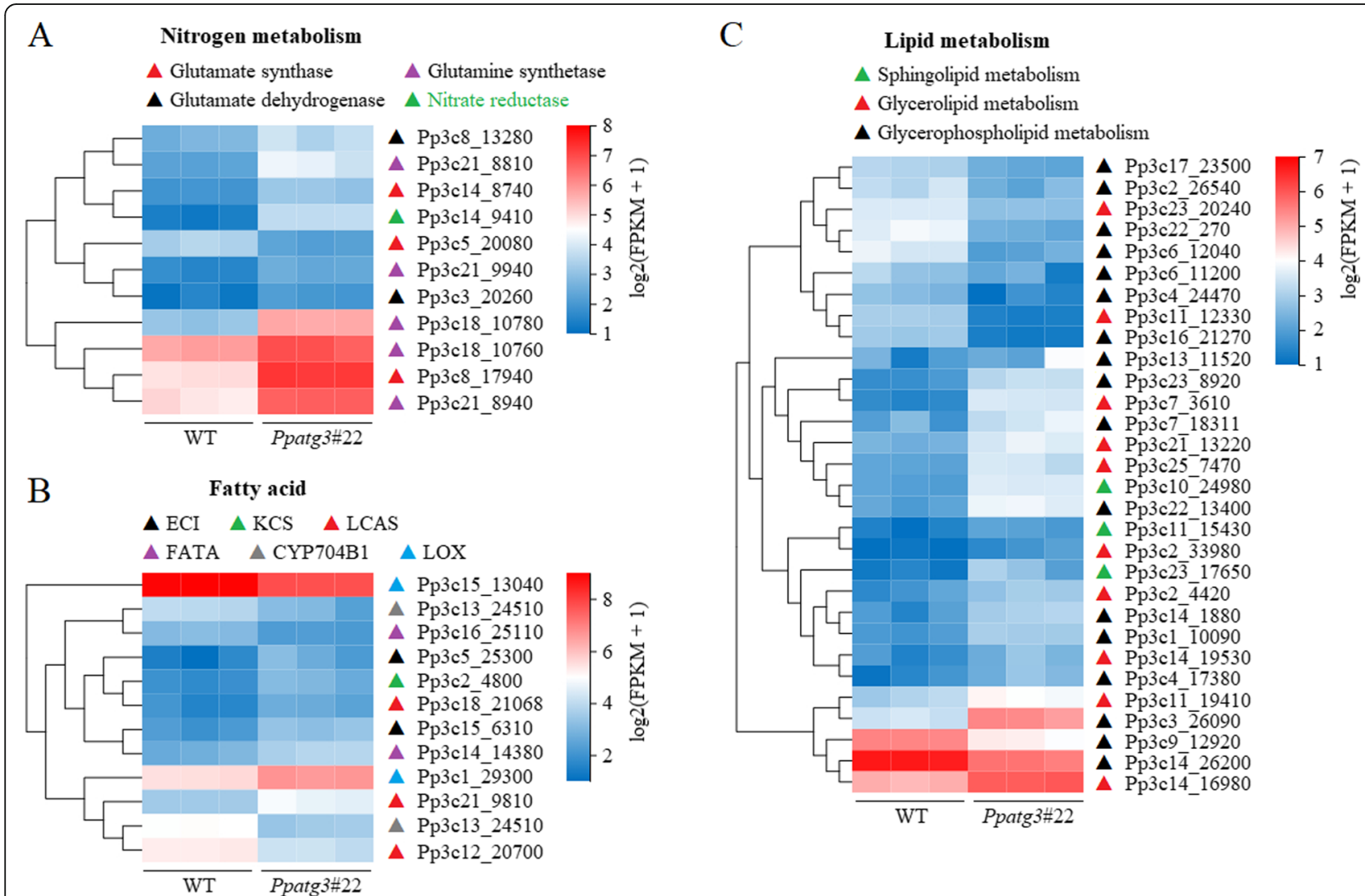

Fig. 5 Differential expression of genes related to nitrogen metabolism and lipid/fatty acid metabolism in Ppatg3 plants. a-c Transcriptional analysis for a subset of genes related to nitrogen metabolism and lipid/fatty acid metabolism in WT and Ppatg3. Expression levels shown as $\log 2($ FPKM+1) values. Three biological replicates were analyzed. Detailed information for each gene is supplied in Additional file 8 


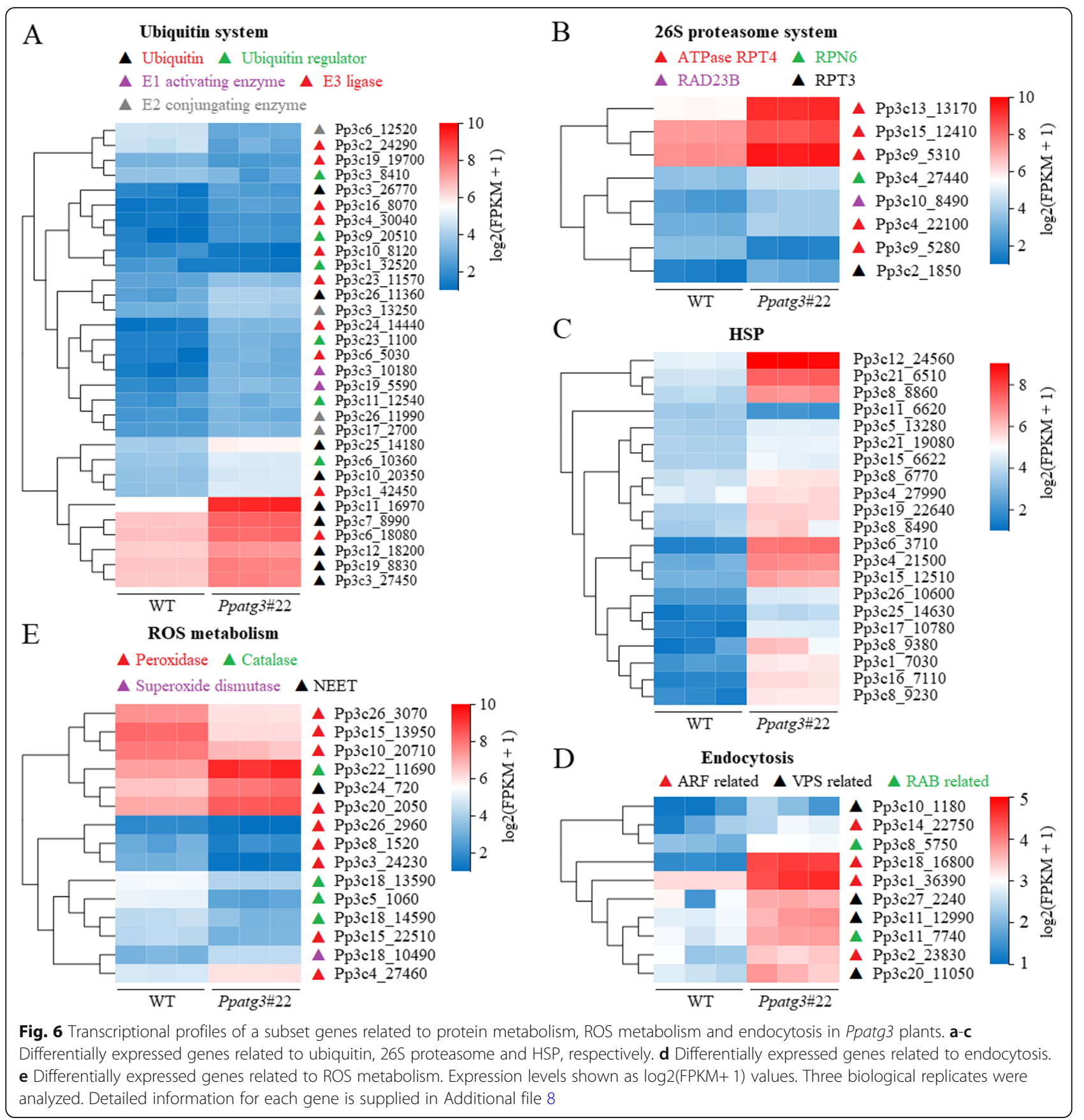

Additional file 8). These results suggest that the activity of the ubiquitin-26S proteasome pathway (UPP) for protein degradation is enhanced in the mutant due to a defect in autophagy.

Heat shock proteins (HSPs) play essential roles in preventing the misfolding of proteins and blocking the formation of large protein aggregates which severely impede cellular functions [36]. The transcript levels of many genes (20 of 21) encoding HSPs/chaperones were significantly higher in Ppatg3 than the WT (Fig. 6c and Additional file 8), suggesting that the resolving of misfolded protein aggregates by HSPs/chaperones was activated in the mutant to maintain the proper protein conformation and extend cell longevity.

Furthermore, 10 genes related to the endocytosis pathway were significantly upregulated in the mutant (Fig. 6d and Additional file 8); this pathway is involved in the recruitment and degradation of cell surface proteins and cellular fatty acids/lipids to support basic cellular functions [37, 38]. However, in contrast to the highly expressed genes related to protein metabolism and endocytosis, the transcript levels of most ROS 
metabolism-related genes (10 of 15) were significantly reduced in the mutant, including 7 and 3 genes encoding peroxidases and catalases, respectively (Fig. 6e and Additional file 8), perhaps leading to the accumulation of ROS and the generation of damaged or toxic materials in the Ppatg3 mutant.

\section{Validation of DEGs by RT-qPCR}

Finally, to validate the gene expression patterns demonstrated by RNA-Seq, we performed RT-qPCR analysis of 21 DEGs, 15 homologous SAGs and 6 genes related to nitrogen metabolism using the same mRNA samples used for RNA-Seq analysis. These genes included several nitrogen metabolism-related genes, including homologs of GLN (Pp3c21_8940, Pp3c21_8810, and Pp3c18_10780 and Pp3c18_10760), GLS (Pp3c8_17940) and NR (Pp3c14_ 9410) (Fig. 7a). We also identified 4 and 11 SAG homologs that were up- and downregulated, respectively, in Ppatg3 knockout plants (Fig. 7b), including homologs of NYE1/2 (Pp3c17_23030), HXK1/GIN2 (Pp3c1_5000, Pp3c19_ 20120, and Pp3c22_9450), PPDK (Pp3c5_22540), ACS10 (Pp3c21_10860), GPR7 (Pp3c7_3360 and Pp3c7_6560), LrgB (Pp3c4_7680), GBF1 (Pp3c21_5770), SID2 (Pp3c9_ 10620), LOX3 (Pp3c15_13040), SAG113 (Pp3c7_5390), CHX24 (Pp3c11_19850) and FTSH5 (Pp3c24_15420). The expression patterns of all genes examined were similar to those obtained by RNA-Seq analysis.

\section{Discussion}

Autophagy is a ubiquitous process that plays important roles in plant development and senescence to maintain essential cellular functions and life activities [3, 7, 19]. Extensive studies have indicated that autophagy is important for $\mathrm{N}$ utilization [26-29], fatty acid/lipid homeostasis [13-15] and the degradation of damaged chloroplasts [16, 17] or aggregated proteins [18] in plants. Although a previous study revealed that autophagy is essential for maintaining the balance of amino acid metabolism in P. patens [30], how this process regulates $\mathrm{C} / \mathrm{N}$ status and fatty acid metabolism in moss has been largely unknown. Here, we demonstrated that the E2-like enzyme PpATG3, which is extensively expressed in tissues (Fig. 1a) and is localized to the cytoplasm (Fig. $1 b)$, is essential for both autophagy and normal plant development in $P$. patens. Thus, Ppatg3 mutant cultured on normal growth medium for 7 to 56 days showed significantly premature senescence of leafy gametophores (Fig. 2a) and reduced new protonema formation (Fig. 2b) compared to WT plants grown under the same conditions.

Early leaf senescence is the principal phenotype of autophagy mutant in Arabidopsis [19, 39]. Thus, we examined several physiological and metabolic markers and performed transcriptome analysis of the Ppatg3 mutant during the appearance of premature senescence in leafy gametophores. After 7 days of culture, yellowing and weak chlorophyll fluorescence were detected in Ppatg3 (Fig. 2a), which is consistent with the significantly reduced $\mathrm{Fv} / \mathrm{Fm}$ values of this mutant (Fig. 2c). After this time point, more serious yellowing was observed, indicating that the Ppatg3 was indeed undergoing premature senescence. Indeed, the chlorophyll contents were significantly lower in the Ppatg3 mutant than in WT plants (Fig. 2d). In addition, Ppatg3 cells appeared hollow and yellow (Fig. 3a and b), which was accompanied by defects in autophagosome formation and led to the accumulation of bulk cytosolic cargos (Fig. 3c and d). These results suggest that physiological defects were present in this autophagy mutant, leading to a prematuresenescence phenotype.

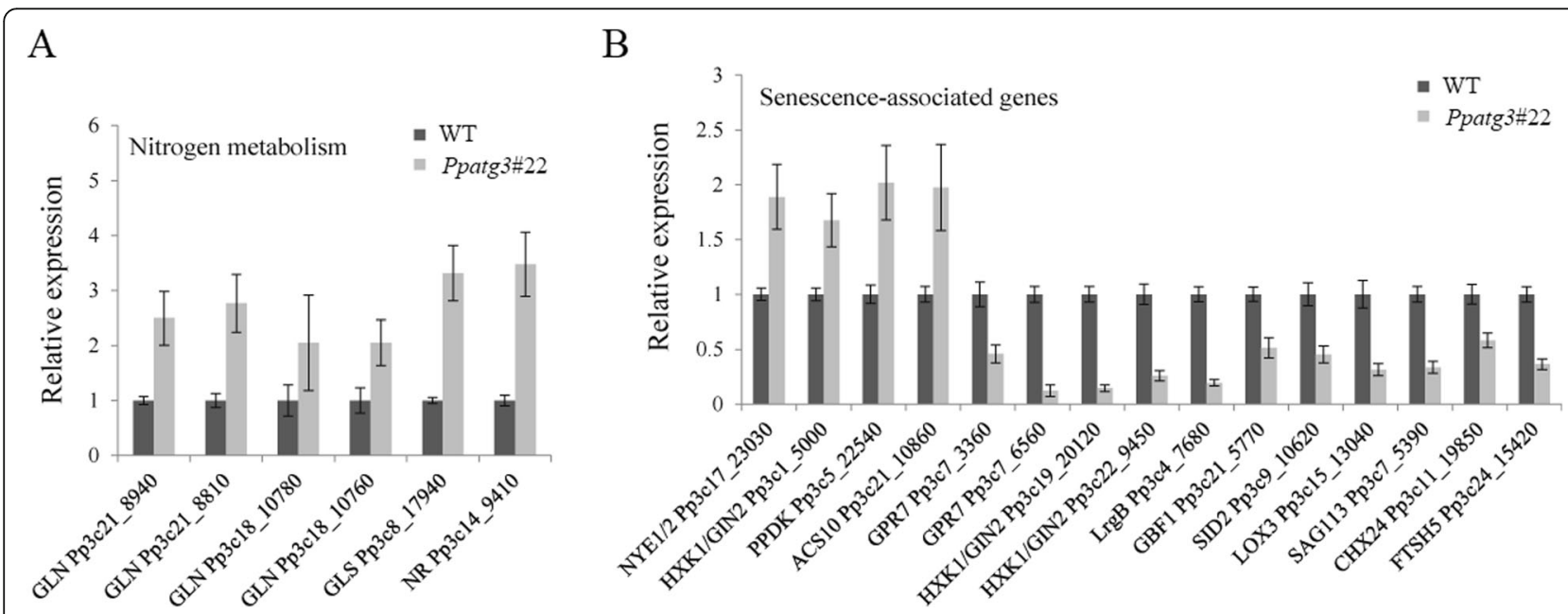

Fig. 7 Transcript abundances of the nitrogen metabolism related genes (a) and SAGs (b) were confirmed by RT-qPCR. Three biological replicates were analyzed and error bars show the mean value \pm SD. The expression value of WT sample was normalized to 1 
The physiological defects in Ppatg3 plants were more severe later in the culture period, at 28 and 56 days. At 14 days, Fv/Fm values and chlorophyll biosynthesis were already significantly reduced in the mutant (Fig. $2 \mathrm{c}$ and d), but there was no significant difference in $\mathrm{N}$ content or $\mathrm{C} / \mathrm{N}$ ratio between $\mathrm{WT}$ and Ppatg3 plants (Fig. $4 \mathrm{a}-\mathrm{c}$ ). By contrast, at 28 days, the Ppatg3 mutant not only exhibited reduced photosynthetic capacity (Fig. 2c and d), but they also displayed dysfunctional nitrogen metabolism resulting in a lower $\mathrm{C} / \mathrm{N}$ ratio (Fig. $4 \mathrm{a}-\mathrm{c}$ ). An autophagy-defective Arabidopsis mutant displays reduced $\mathrm{N}$ levels, resulting in a higher $\mathrm{C} / \mathrm{N}$ ratio [29]. By contrast, the lower $\mathrm{C} / \mathrm{N}$ ratio in the Ppatg3 mutant relative to WT plants was clearly linked to dysfunctional nitrogen metabolism at both 28 and 56 days (Fig. $4 \mathrm{a}-\mathrm{c}$ ). These results indicate that the effects of autophagy on nitrogen metabolism differ between $P$. patens and $A$. thaliana.

Chloroplast PGs are lipoprotein particles with a high lipid-to-protein ratio that function in chloroplast biogenesis whose numbers increase during the process of plant senescence [33]. Estimates suggest that $75-80 \%$ of the total nitrogen content in a plant leaf is stored in chloroplasts [40]. In the current study, we observed irregular chloroplasts with a high density of PGs in leafy gametophore cells of Ppatg3 but not WT plants (Fig. 3e-f). The defects in chlorophyll biosynthesis and $\mathrm{N}$ metabolism in the mutant might have contributed to its defective chloroplast development. Chloroplasts with accumulated PGs in Arabidopsis were regular during normal senescence [33]; however, the chloroplasts that accumulated PGs in Ppatg3 appeared damaged, with an irregular shape. Perhaps a defect in autophagy in the Ppatg3 mutant impaired the degradation and turnover process of unnecessary or damaged chloroplasts, leading to the accumulation of PGs and abnormally shaped chloroplasts, thereby resulting in reduced chlorophyll levels.

Autophagy plays important roles in lipid/fatty acid metabolism, composition and turnover in several vascular plants, such as Arabidopsis and maize [13, 14]. Moreover, in rice, the metabolism of lipids such as triacylglycerols (TAGs) and diacylglycerols (DAGs) was impaired during pollen maturation in an autophagy mutant [11]. Fatty acids are important components of TAGs and DAGs [41]; however, the potential role of autophagy in fatty acid metabolism in moss has not been reported. In the current study, we discovered that the loss of autophagy affected the relative levels of six fatty acids, resulting in their impaired metabolism (Fig. $4 \mathrm{~d}-\mathrm{e}$ ). In particular, the relative levels of linoleic acid (18:2) and arachidonic acid (20:4) were sharply reduced in the Ppatg3 mutant. Supplementation with linoleic acid and arachidonic acid together, but not individually, restored the $\mathrm{C} / \mathrm{N}$ ratio of the mutant compared to the WT (Additional file 2A-C), suggesting that the disrupted $\mathrm{N}$ metabolism in the mutant might be related to the reduction in fatty acid contents. These results suggest that the early-senescence phenotype of Ppatg3 was partially caused by impaired $\mathrm{N}$ utilization and an imbalance in fatty acid contents. However, the premature gametophore senescence was not relieved by fatty acid supplementation (Additional file 2D), suggesting that autophagy-dependent senescence is influenced by other factors besides the $\mathrm{C} / \mathrm{N}$ ratio and fatty acid metabolism, an issue worthy of further study.

During the normal senescence process, fatty acids accumulate in chloroplast PGs and chlorophylls and lipids are extensively degraded [42]. In rice, senescence is accompanied by an increase in the number of chloroplast PGs and significant changes in the contents of fatty acids, especially increases in palmitic acid (16:0) levels [43]. Similarly, in the current study, we observed that the Ppatg3 mutant contained an unusually high density of chloroplast PGs (Fig. 3e and f) and high palmitic acid (16:0) contents (Fig. 4d and e), which is consistent with previous reports. In addition, the Ppatg3 mutant contained significantly higher levels of another fatty acid, arachidic acid (20:0), compared to the WT in both 28and 56-day-old plants (Fig. 4d and e). However, the levels of two fatty acids, stearic acid (18:0) and oleic acid (18:1), behaved differently at 28 vs. 56 days (Fig. $4 d$ and e). These results suggest that changes in fatty acid contents during autophagy-dependent senescence might also be related to PG accumulation in chloroplasts, which might have partially contributed to the physiological defects of the autophagy mutant.

Additionally, RNA-Seq revealed that the loss of ATG3 in $P$. patens significantly altered transcript abundance/ gene expression (Additional file 3). Analysis of the top 20 enriched KEGG pathways of the DEGs suggested that biosynthetic and metabolic pathways were seriously affected in the mutant (Additional file 3D-E and Additional file 6). As expected, the nitrogen metabolism pathway was significantly enriched (Additional file 3D) by the differential expression of a subset of nitrogenrelated genes (Fig. 5a), which might be related to the $\mathrm{N}$ contents of the Ppatg3 mutant. Furthermore, many genes involved in fatty acid/lipid metabolism were significantly differentially expressed in the mutant vs. the WT (Fig. 5b and c), which might be associated with the changes in fatty acid contents and premature senescence due to defective autophagy. Dysfunctional autophagy led to the increased transcription of genes associated with UPP and HSP, a phenomenon that also occurs in endocytosis-related genes (Fig. 6a-d). These findings suggest that the degradation of unnecessary proteins or fatty acids/lipids is promoted in the Ppatg3 mutant for recycling to support basic cellular functions and extend longevity. However, a subset of ROS metabolism-related 
genes was significantly downregulated in the mutant (Fig. 6e and Additional file 8), which might have contributed to senescence resulting from damaged or toxic materials generated by ROS accumulation in Ppatg3.

As described in Arabidopsis [35], numerous genes related to SAGs, chlorophyll biosynthesis and photosystems were differentially expressed in Ppatg3 vs. the WT plants, suggesting that the senescence process began early in the Ppatg3 mutant (Additional file 7). For example, the $P$. patens homologs of NYE1/2 and PPDK were upregulated in the Ppatg3 mutant, as evidenced by both RNA-Seq analysis and RT-qPCR validation. NYE1/ 2 and $P P D K$ encode important regulators of chlorophyll degradation and nitrogen remobilization, respectively, that function during green leaf senescence in Arabidopsis [44-46] (Additional file 7 and Fig. 7b). These results suggest that the chlorophyll degradation and nitrogen utilization mechanisms might be conserved to some degree between autophagy-defective senescence and normal green leaf senescence. However, several $P$. patens SAG homologs, such as SAG113, were downregulated in the Ppatg3 mutant (Additional file 7 and Fig. 7b), pointing to possible differences in the regulatory mechanisms of autophagy-defective senescence vs. normal green leaf senescence.

A recent study in yeast revealed that the ATG3 allosteric activation switch element E123IR plays essential roles in removing intramolecular interactions of ATG3 with the E1-like enzyme ATG7 and E3-like enzyme ATG12-ATG5 complex to remodel the active site of ATG3, thereby mediating the lipidation reaction of ATG8-PE during the autophagy process [47]. As expected, $\mathrm{Y} 2 \mathrm{H}$ assays demonstrated that PpATG3 strongly interacts with PpATG7 and PpATG12 in P. patens (Additional file 9), suggesting that the interaction network of ATG3 with ATG7 and ATG12 might be conserved. However, whether the exact interaction mechanism of ATG3 with E1- or E3-like enzymes in plants is identical to that discovered in yeast requires further study.

Taken together, these results indicate that the premature-senescence phenotype caused by Ppatg3 knockout is influenced by a series of complex metabolic pathways, including $\mathrm{N}$ utilization, fatty acid/lipid metabolism, damaged chloroplast degradation, ROS removal and the recycling of unnecessary proteins due to the disruption of autophagy. However, the exact mechanisms underlying the roles of autophagy in these physiological defects should be more fully elucidated in the future.

\section{Conclusions}

Our analysis of $P$. patens, an emerging model system for autophagy research, provided new insights into the role of autophagy in preventing plant senescence. Our results support the role of PpATG3 in autophagosome formation. Autophagy-defective mutant due to a PpATG3 deletion showed premature gametophore senescence under non-stress conditions, which could be partially explained by impaired $\mathrm{N}$ metabolism. We also provided evidence for the role of autophagy in fatty acid metabolism. The Ppatg3 mutant showed reduced levels of linoleic acid and arachidonic acid and a concomitant increase in the levels of palmitic acid and arachidic acid. Supplementation with linoleic acid and arachidonic acid together restored the $\mathrm{N}$ content but not the premature senescence of the mutant, suggesting that a more complex mechanisms contributes to autophagy-dependent longevity. Our results also suggest that the differential expression of numerous biosynthetic and metabolic pathway genes played a role in the premature senescence of the Ppatg3 mutant due to defective autophagy. These results suggest that PpATG3 directly or indirectly affects $\mathrm{C} / \mathrm{N}$ stability and fatty acid metabolism, as well as numerous other biosynthetic and metabolic pathways, such as damaged chloroplast degradation and unnecessary protein recycling, by functioning in autophagy under normal growth conditions, thus preventing premature senescence and extending plant longevity.

\section{Methods}

Plant material, growth condition and moss transformation Physcomitrella patens Gransden wild-type plants were supplied by Prof. Mitsuyasu Hasebe, Japan National Institute for Basic Biology (NIBB). Plants were grown on BCD medium supplemented with $5 \mathrm{mM}$ ammonium tartrate and $1 \mathrm{mM} \mathrm{CaCl}_{2}$ [48] at $25^{\circ} \mathrm{C}$ under a photoperiod of $16 \mathrm{~h}$ light with a photon flux of 60 to $80 \mu \mathrm{mol} \mathrm{m}^{-2} \mathrm{~s}^{-1}$ for 1 to 8 weeks. To generate protonemal tissues, plant material was crushed with an ULTRA-TURRAX ${ }^{\circ}$ Tube Drive in $5 \mathrm{ml}$ of sterile water and was propagated onto BCD medium. Cultures were maintained by inoculating a small explant of seven-day-old protonemal tissue (1-2 $\mathrm{mm}$ ) using forceps to fresh culture medium. Polyethylene glycol (PEG)-mediated transformation of protoplasts for gene transient expression or gene stable deletion was described previously by Shi and Theg [49].

\section{Protein sequence alignment, motif and phylogenetic analysis}

The full-length protein sequences of the PpATG3 (Pp3c8_11900), KnATG3 (GAQ83284), MeATG3 (ME000344S06021), AaATG3 (AANG000634), MpATG3 (Mapoly0003s0208), BdATG3 (Bradi3g33350) AtATG3 (AT5G61500), MmATG3 (NP_080678), HsATG3 (NP_ 071933) and ScATG3 (YNR007C) were obtained from Phytozome, NCBI and SGD database, respectively. ALN file was obtained by multiple protein sequences alignment using ClustalX2.0 [50], and the crystal structure of 
ScATG3 (PDB ID: 2DYT) [21] was used for secondary structure depiction by web tool ESPript 3.0 [51]. The domain and motif searching were performed by SMART [52] and MEME SUITE [53], respectively. The phylogenetic tree was carried out by MEGA 6 [54] using a neighbour-joining (NJ) method.

\section{Microarray expression analysis}

Microarray expression values in different tissues of PpATG3 gene was analyzed basing previous microarray data [32] of $P$. patens. Ten tissues were obtained for analysis including protonemata (chloronema and caulonema), gametophore, archegonia, four sporophyte developing stages (S1, S2, S3 and M), spores, and rooting structure rhizoids.

\section{Subcellular localization of PpATG3}

Full length coding sequence (lacking the stop codon) of PpATG3 was PCR amplified from P. patens cDNA template using the primers PpATG3-F and PpATG3$\mathrm{R}$ (Additional file 10). The resulting 921-bp PCR fragment was cloned using KpnI and XbaI restriction enzymes (Thermo Scientific FastDigest, FD0524 and FD0684) into the vector pM999 [55] by sticky end ligation. The pM999 contains the eGFP coding sequence driven by a $35 \mathrm{~S}$ promoter. The chimeric gene p35S:PpATG3-eGFP was constructed by fusing the PpATG3 cDNA sequence to the $5^{\prime}$ end frame of eGFP. After this, the resulting plasmid was introduced into the protoplast of $P$. patens by PEG-mediated transformation [48]. $48 \mathrm{~h}$ after culture at $25^{\circ} \mathrm{C}$, protoplasts were observed by a confocal microscopy for GFP fluorescence.

\section{PpATG3 gene disruption}

The $5^{\prime}$ and $3^{\prime}$ flanking regions $(863 \mathrm{bp}$ and $800 \mathrm{bp}$ respectively) of the PpATG3 gene were amplified using 2 primer pairs $\mathrm{P} 1 / \mathrm{P} 2$ and $\mathrm{P} 3 / \mathrm{P} 4$, respectively. Two fragments were digested with $\mathrm{KpnI} / \mathrm{HindIII}$ and $\mathrm{XbaI} /$ BamHI respectively (Thermo Scientific FastDigest, FD0524, FD0504, FD0684 and FD0054), and cloned by sequential into the pTN182 vector upstream and downstream of the nptII gene driven by a Pm35S promoter (geneticin resistance gene cassettes) (http://moss.nibb.ac. $\mathrm{jp} /$ ). Construct was linearized with $\mathrm{KpnI}$ and BamHI prior to transformation. Transformation was completed as described previously [48]. The primers for genotyping the PpATG3 gene knockout were used can be found in Additional file 10. PpUbiquitin [56] and PpAdePRT [57] was used to assess DNA and cDNA template quality, respectively.

\section{Analysis of chlorophyll fluorescence and chlorophyll content}

Chlorophyll florescence values were monitored with an IMAGING-PAM FluorImager and Imaging Win software. Prior to determine Fv/Fm, the cultures were dark adaptation for minimum $30 \mathrm{~min}$. Chlorophyll were isolated from cultures using $\operatorname{DMF}(\mathrm{N}, \mathrm{N}-$ dimethylformamide) as described previously [58]. Absorbance was measured at 647 and 664 of the supernatants using DMF as blank. Total chlorophyll content (chlorophyll a + chlorophyll b) was calculated using the formula Chlorophyll a $(\mathrm{mmol} / \mathrm{g})=[(12 \times \mathrm{A} 664-3.11 \times$ A647) $\times \mathrm{mL}$ DMF] / mg Dry Weight and Chlorophyll b $(\mathrm{mmol} / \mathrm{g})=[(20.78 \times \mathrm{A} 647-4.88 \times \mathrm{A} 664) \times \mathrm{mL} \quad \mathrm{DMF}] \quad /$ mg Dry Weight.

\section{Phenotypic observation}

For recording phenotypic characterization of WT and Ppatg3 plants, cultures were grown for 7 to 56 days under normal conditions. Leafy gametophores were observed by light microscopy. TEM was used to observe the Ppatg3 mutant. Gametophores of 28-day-old WT and Ppatg3 plants were fixed overnight in 3\% glutaraldehyde and then postfixed for $2 \mathrm{~h}$ in $1 \%$ osmium tetroxide (OsO4), dehydrated in a serial ethanol gradient and embedded in Epon 812 resin. Samples were fixed at $4{ }^{\circ} \mathrm{C}$. Serial and uniform-thickness of sections about $70 \mathrm{~nm}$ of gametophores leafy cells were generated by a Leica EM UC7 ultramicrotome. Sections were carried onto 100mesh $\mathrm{Cu}$ grids, and then stained sequentially by $2 \%$ uranyl acetate solution and lead citrate. Afterward, the sections were observed in microscope JEM 1400Plus at 120, $000 \mathrm{~V}$. To detect autophagosomes of WT and Ppatg3 mutant, 28-day-old plants were treated by $100 \mathrm{mM} \mathrm{NaCl}$ for $1 \mathrm{~h}$ and then used for TEM observation.

\section{Measurement of carbon and nitrogen content}

Plants were collected and dried $10 \mathrm{~h}$ at $100{ }^{\circ} \mathrm{C}$ in a drying oven. Then the subsamples were ground into a homogenous fine powder and carefully measured in tin capsules by a fully automatic $\mathrm{C}, \mathrm{N}$ analyzer Elementar vario Micro cube to establish carbon and nitrogen content.

\section{Fatty acid content analysis}

In this study, $1 \mathrm{~g}$ moss tissue of WT and Ppatg3 plants were used for lipid extraction. Then the samples were utilized for methyl esterification and detailed method was performed as previously described [34]. Fatty acid contents were measured by a GC-MS analyzer Agilent Technologies 7890A GC system. Peak identification and fatty acid consideration were performed with the MSD ChemStation software. 
Fatty acid supplementation

For fatty acid supplementation, 21-day-old WT and Ppatg3 plants grown on normal BCD medium were transferred to BCD medium with $20 \mu \mathrm{g} / \mathrm{ml}$ linoleic acid (Shanghai yuanye, DC14635400) or $20 \mu \mathrm{g} / \mathrm{ml}$ arachidonic acid (Shanghai yuanye, B20540) for 7 days, or both with linoleic acid and arachidonic acid at the same concentration for 7 days. As a control, 21-day-old WT and Ppatg3 plants grown on normal BCD medium were transferred to $\mathrm{BCD}$ medium with $0.1 \%$ DMSO for 7 days.

\section{RNA-sequencing and data analysis}

WT and Ppatg3 plants were grown on BCD medium for 28d. Total RNA of the whole plant was extracted by using a RnaExTM solution (Generay). cDNA library construction, sequencing and data analysis were carried out by the BGISEQ-500 platform established by BGITech (Wuhan, China). The reads alignment and expression level calculation were conducted by using Bowtie 2 [59] and RSEM [60], respectively. A differential expression transcript/gene with at least two-fold change and $p$ value $\leq 0.001$ was used as further analysis [61]. $R$ program "princomp", "phyper" and "pheatmap" were used to conduct PCA analysis, KEGG pathway enrichment and construction of heat maps, respectively. The RNASeq data of the present study had been deposited at the BIG genome sequence archive (GSA) under Bioproject identifier PRJCA001964 with accession number: SAMC116811 to SAMC116814.

\section{RNA isolation and real-time quantitative PCR}

RnaExTM solution (Generay) was used for total RNA extraction. First-strand cDNA was synthesized by a reverse transcription kit (Transgen). Real-Time quantitative PCR (RT-qPCR) was performed as previously described [62]. The primers for gene expression analysis were used in this study can be found in Additional file 10. The relative expression levels were calculated using PpAdePRT [57] as expression control.

\section{$\mathrm{Y} 2 \mathrm{H}$ analysis}

To determine the protein interactions between PpATG3 and PpATG7 (Pp3c24_8100) or PpATG12 (Pp3c4_ 29920), we performed the $\mathrm{Y} 2 \mathrm{H}$ assays (Clontech). In our study, the CDS of PpATG3 gene was amplified and cloned into bait vector pGBKT7 (BD), while the CDS of PpATG7 or PpATG12 gene was amplified and cloned into prey vector pGADT7 (AD). The $\mathrm{Y} 2 \mathrm{H}$ assays were carried out by a Frozen-EZ Yeast Transformation II Kit (Zymo Research). The primers for $\mathrm{Y} 2 \mathrm{H}$ assays in this study were listed in Additional file 10.

\section{Supplementary information}

Supplementary information accompanies this paper at https://doi.org/10. 1186/s12870-020-02651-6.

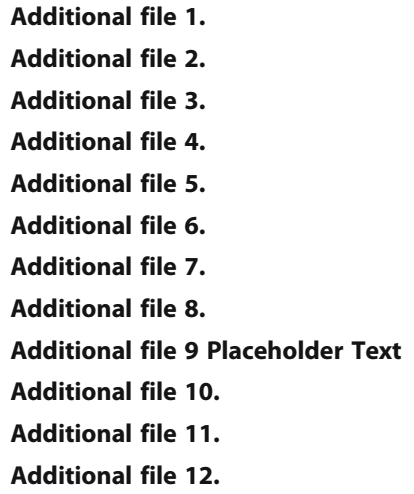

Additional file 1.

Additional file 2.

Additional file 3.

Additional file 4

Additional file 5 .

Additional file 6 .

Additional file 7.

Additional file 8.

Additional file 9 Placeholder Text

Additional file 10.

Additional file 11.

Additional file 12.

\section{Abbreviations}

ATG: AuTophaGy; Cys: Cysteine; SAGs: Senescence-associated genes; WT: Wildtype; PGs: Plastoglobuli; ROS: Reactive oxygen species; UPP: Ubiquitin-26S proteasome pathway; DETs: Differentially expressed transcripts;

DEGs: Differentially expressed genes; FPKM: Fragments per kilobase of transcript per million fragments mapped; KEGG: Kyoto encyclopedia of genes and genomes; TEM: Transmission electron microscopy; RT-qPCR: Real-Time quantitative PCR

\section{Acknowledgements}

We thank Prof. Mitsuyasu Hasebe for providing the moss spores and pTN182 plasmid, and we also thank Prof. Jianqiang Wu for providing the pM999 plasmid.

Authors' contributions

ZC and WW contributed equally to this work. ZC and WW performed the experiments, wrote the manuscript and analyzed the data. XP, XD, and PL worked on moss transformation. $L L$ designed the experiment, supervised the research, and revised the manuscript. All authors discussed the project and approved the manuscript.

\section{Funding}

This work was supported by the CAS Pioneer Hundred Talents Program, the National Natural Science Foundation of China (No. 31971410) and the postdoctoral Fund of Yunnan Province (No. Y732681261). The funding body played no role in the design of the study, the collection, analysis and interpretation of the data and in writing the manuscript.

\section{Availability of data and materials}

All data generated or analyzed in this study are included in this article and the additional files. Original files for figures can be found in Additional files 11 and 12. Other data are available from the corresponding author on reasonable request. The raw RNA-Seq data were submitted to the GSA database with the bioproject ID: PRJCA001964, with accession number SAMC116811 to SAMC116814 (https://bigd.big.ac.cn/gsa/).

Ethics approval and consent to participate

Not applicable.

Consent for publication

Not applicable.

\section{Competing interests}

The authors declare that they have no competing interests.

\section{Author details}

${ }^{1}$ Department of Economic Plants and Biotechnology, Yunnan Key Laboratory for Wild Plant Resources, Kunming Institute of Botany, Chinese Academy of Sciences, Kunming 650204, China. ${ }^{2}$ University of the Chinese Academy of 
Sciences, Beijing 100049, China. ${ }^{3}$ Sino-Danish College, University of Chinese Academy of Sciences, Beijing 100049, China. ${ }^{4}$ State Key Laboratory of Agrobiotechnology, The Chinese University of Hong Kong, Hong Kong 999077, China. ${ }^{5}$ Germplasm Bank of Wild Species, Kunming Institute of Botany, Chinese Academy of Sciences, Kunming 650204, China. ${ }^{6}$ Key Laboratory for Forest Resources Conservation and Utilization in the Southwest Mountains of China Ministry of Education, Southwest Forestry University, Kunming 650204, China. ${ }^{7}$ State Key Laboratory of Biocatalysis and Enzyme Engineering, Hubei Collaborative Innovation Center for Green Transformation of Bio-Resources, Hubei Key Laboratory of Industrial Biotechnology, School of Life Sciences, Hubei University, Wuhan 430062, China.

Received: 12 March 2020 Accepted: 15 September 2020 Published online: 23 September 2020

\section{References}

1. Yang Z, Klionsky DJ. Eaten alive: a history of macroautophagy. Nat Cell Biol. 2010;12:814-22.

2. Avila-Ospina L, Moison M, Yoshimoto K, Masclaux-Daubresse C. Autophagy, plant senescence, and nutrient recycling. J Exp Bot. 2014;65:3799-811.

3. Üstün S, Hafrén A, Hofius D. Autophagy as a mediator of life and death in plants. Curr Opin Plant Biol. 2017;40:122-30.

4. Masclaux-Daubresse $\mathrm{C}$, Chen $\mathrm{Q}$, Havé $\mathrm{M}$. Regulation of nutrient recycling via autophagy. Curr Opin Plant Biol. 2017;39:8-17.

5. Liao CY, Bassham DC. Combating stress: the interplay between hormone signaling and autophagy in plants. J Exp Bot. 2020;71:1723-33.

6. Wang P, Mugume Y, Bassham DC. New advances in autophagy in plants: regulation, selectivity and function. Semin Cell Dev Biol. 2018;80:113-22.

7. Bassham DC, Laporte M, Marty F, et al. Autophagy in development and stress responses of plants. Autophagy. 2006;2(1):2-11.

8. Xie Z, Klionsky DJ. Autophagosome formation: core machinery and adaptations. Nat Cell Biol. 2007:9:1102-9.

9. Liu Y, Xiong Y, Bassham DC. Autophagy is required for tolerance of drought and salt stress in plants. Autophagy. 2009:5:954-63.

10. Daudi A, Cheng Z, O'Brien JA, et al. The apoplastic oxidative burst peroxidase in Arabidopsis is a major component of pattern-triggered immunity. Plant Cell. 2012;24:275-87.

11. Kurusu T, Koyano T, Hanamata S, et al. OsATG7 is required for autophagydependent lipid metabolism in rice postmeiotic anther development. Autophagy. 2014;10:878-88.

12. Wang $Y$, Yu B, Zhao J, et al. Autophagy contributes to leaf starch degradation. Plant Cell. 2013;25:1383-99.

13. Havé M, Luo J, Tellier F, et al. Proteomic and lipidomic analyses of the Arabidopsis atg 5 autophagy mutant reveal major changes in endoplasmic reticulum and peroxisome metabolisms and in lipid composition. New Phytol. 2019;223:1461-77.

14. McLoughlin F, Augustine RC, Marshall RS, et al. Maize multi-omics reveal roles for autophagic recycling in proteome remodelling and lipid turnover. Nat Plants. 2018;4:1056-70.

15. Masclaux-Daubresse C, d'Andrea S, Bouchez I, Cacas JL. Reserve lipids and plant autophagy. J Exp Bot. 2020;71:2854-61.

16. Otegui MS. Vacuolar degradation of chloroplast components: autophagy and beyond. J Exp Bot. 2018;69:741-50.

17. Woodson JD. Chloroplast stress signals: regulation of cellular degradation and chloroplast turnover. Curr Opin Plant Biol. 2019:52:30-7.

18. Yu L, Chen Y, Tooze SA. Autophagy pathway: cellular and molecular mechanisms. Autophagy. 2018;14:207-15.

19. Hanaoka H, Noda T, Shirano Y, et al. Leaf senescence and starvationinduced chlorosis are accelerated by the disruption of an Arabidopsis autophagy gene. Plant Physiol. 2002;129:1181-93.

20. Ichimura Y, Kirisako T, Takao T, et al. A ubiquitin-like system mediates protein lipidation. Nature. 2000;408:488-92.

21. Yamada Y, Suzuki NN, Hanada T, et al. The crystal structure of Atg3, an autophagy-related ubiquitin carrier protein (E2) enzyme that mediates Atg8 lipidation. J Biol Chem. 2007;282:8036-43.

22. Sakoh-Nakatogawa M, Kirisako H, Nakatogawa H, Ohsumi Y. Localization of Atg3 to autophagy-related membranes and its enhancement by the Atg8family interacting motif to promote expansion of the membranes. FEBS Lett. 2015;589:744-9.
23. Besteiro S, Brooks CF, Striepen B, Dubremetz JF. Autophagy protein Atg3 is essential for maintaining mitochondrial integrity and for normal intracellular development of Toxoplasma gondii tachyzoites. PLoS Pathog. 2011;7(12): e1002416.

24. Han S, Wang Y, Zheng X, et al. Cytoplastic glyceraldehyde-3-phosphate dehydrogenases interact with ATG3 to negatively regulate autophagy and immunity in Nicotiana benthamiana. Plant Cell. 2015;27:1316-31.

25. Liu Y, Bassham DC. Autophagy: pathways for self-eating in plant cells. Annu Rev Plant Biol. 2012:63:215-37.

26. Guiboileau A, Yoshimoto K, Soulay F, Bataillé MP, Avice JC, MasclauxDaubresse C. Autophagy machinery controls nitrogen remobilization at the whole-plant level under both limiting and ample nitrate conditions in Arabidopsis. New Phytol. 2012:194:732-40.

27. Chen Q, Shinozaki D, Luo J, et al. Autophagy and nutrients management in plants. Cells. 2019:8:1426.

28. Marshall RS, Vierstra RD. Autophagy: the master of bulk and selective recycling. Annu Rev Plant Biol. 2018:69:173-208.

29. Guiboileau A, Avila-Ospina L, Yoshimoto K, et al. Physiological and metabolic consequences of autophagy deficiency for the management of nitrogen and protein resources in Arabidopsis leaves depending on nitrate availability. New Phytol. 2013;199:683-94.

30. Mukae K, Inoue Y, Moriyasu Y. ATG5-knockout mutants of Physcomitrella provide a platform for analyzing the involvement of autophagy in senescence processes in plant cells. Plant Signal Behav. 2015;10(11): e1086859.

31. Sanchez-Vera V, Kenchappa CS, Landberg K, et al. Autophagy is required for gamete differentiation in the moss Physcomitrella patens. Autophagy. 2017; 13:1939-51.

32. Ortiz-Ramírez C, Hernandez-Coronado M, Thamm A, et al. A transcriptome atlas of Physcomitrella patens provides insights into the evolution and development of land plants. Mol Plant. 2016;9:205-20.

33. van Wijk KJ, Kessler F. Plastoglobuli: plastid microcompartments with integrated functions in metabolism, plastid developmental transitions, and environmental adaptation. Annu Rev Plant Biol. 2017:68:253-89.

34. Beike AK, Jaeger C, Zink F, Decker EL, Reski R. High contents of very longchain polyunsaturated fatty acids in different moss species. Plant Cell Rep. 2014;33:245-54

35. Wang X, Gao J, Gao S, Song Y, Yang Z, Kuai B. The H3K27me3 demethylase REF6 promotes leaf senescence through directly activating major senescence regulatory and functional genes in Arabidopsis. PLoS Genet. 2019;15(4):e1008068.

36. Jacob P, Hirt H, Bendahmane A. The heat-shock protein/chaperone network and multiple stress resistance. Plant Biotechnol J. 2017:15:405-14

37. Fan L, Li R, Pan J, Ding Z, Lin J. Endocytosis and its regulation in plants. Trends Plant Sci. 2015:20:388-97.

38. Jaishy B, Abel ED. Lipids, lysosomes, and autophagy. J Lipid Res. 2016;57: 1619-35.

39. Doelling JH, Walker JM, Friedman EM, Thompson AR, Vierstra RD. The APG8/ 12-activating enzyme $A P G 7$ is required for proper nutrient recycling and senescence in Arabidopsis thaliana. J Biol Chem. 2002;277:33105-14.

40. Makino A, Osmond B. Effects of nitrogen nutrition on nitrogen partitioning between chloroplasts and mitochondria in pea and wheat. Plant Physiol. 1991:96:355-62.

41. Durrett TP, Benning C, Ohlrogge J. Plant triacylglycerols as feedstocks for the production of biofuels. Plant J. 2008:54:593-607.

42. Tevini M, Steinmüller D. Composition and function of plastoglobuli: II. Lipid composition of leaves and plastoglobuli during beech leaf senescence. Planta. 1985;163:91-6.

43. Zhang MP, Zhang CJ, Yu GH, et al. Changes in chloroplast ultrastructure, fatty acid components of thylakoid membrane and chlorophyll a fluorescence transient in flag leaves of a super-high-yield hybrid rice and its parents during the reproductive stage. J Plant Physiol. 2010;167:277-85.

44. Ren $G, A n K$, Liao $Y$, et al. Identification of a novel chloroplast protein AtNYE1 regulating chlorophyll degradation during leaf senescence in Arabidopsis. Plant Physiol. 2007;144:1429-41.

45. Taylor L, Nunes-Nesi A, Parsley K, et al. Cytosolic pyruvate, orthophosphate dikinase functions in nitrogen remobilization during leaf senescence and limits individual seed growth and nitrogen content. Plant J. 2010;62:641-52.

46. Wu S, Li Z, Yang L, et al. NON-YELLOWING2 (NYE2), a close Paralog of NYE1, plays a positive role in chlorophyll degradation in Arabidopsis. Mol Plant. 2016;9:624-7. 
47. Zheng Y, Qiu Y, Grace CRR, Liu X, Klionsky DJ, Schulman BA. A switch element in the autophagy E2 Atg3 mediates allosteric regulation across the lipidation cascade. Nat Commun. 2019;10:3600.

48. Liu L, McNeilage RT, Shi LX, Theg SM. ATP requirement for chloroplast protein import is set by the $\mathrm{km}$ for ATP hydrolysis of stromal Hsp70 in Physcomitrella patens. Plant Cell. 2014;26:1246-55.

49. Shi LX, Theg SM. A stromal heat shock protein 70 system functions in protein import into chloroplasts in the moss Physcomitrella patens. Plant Cell. 2010;22:205-20.

50. Larkin MA, Blackshields G, Brown NP, et al. Clustal W and Clustal X version 2. 0. Bioinformatics. 2007;23:2947-8.

51. Robert $X$, Gouet P. Deciphering key features in protein structures with the new ENDscript server. Nucleic Acids Res. 2014;42:W320-4.

52. Letunic I, Bork P. 20 years of the SMART protein domain annotation resource. Nucleic Acids Res. 2018:46:D493-6.

53. Bailey TL, Boden M, Buske FA, et al. MEME SUITE: tools for motif discovery and searching. Nucleic Acids Res. 2009:37:W202-8.

54. Tamura K, Stecher G, Peterson D, Filipski A, Kumar S. MEGA6: molecular evolutionary genetics analysis version 6.0. Mol Biol Evol. 2013;30:2725-9.

55. Pu X, Yang L, Liu L, et al. Genome-wide analysis of the MYB transcription factor superfamily in Physcomitrella patens. Int J Mol Sci. 2020;21:975.

56. Arya D, Kapoor S, Kapoor M. Physcomitrella patens DNA methyltransferase 2 is required for recovery from salt and osmotic stress. FEBS J. 2016;283:55670.

57. Le Bail A, Scholz S, Kost B. Evaluation of reference genes for RT qPCR analyses of structure-specific and hormone regulated gene expression in Physcomitrella patens gametophytes. PLoS One. 2013;8:e70998.

58. Suzuki R, Ishimaru T. An improved method for the determination of phytoplankton chlorophyll using $\mathrm{N}$, N-dimethylformamide. Journal of the Oceanographical Society of Japan. 1990;46:190-4.

59. Langmead B, Salzberg SL. Fast gapped-read alignment with bowtie 2. Nat Methods. 2012;9:357-9.

60. Li B, Dewey CN. RSEM: accurate transcript quantification from RNA-Seq data with or without a reference genome. BMC Bioinformatics. 2011;12:323.

61. Wang L, Feng Z, Wang X, Wang X, Zhang X. DEGseq: an R package for identifying differentially expressed genes from RNA-seq data. Bioinformatics. 2010;26:136-8.

62. Xu W, Chen Z, Ahmed N, Han B, Cui Q, Liu A. Genome-wide identification, evolutionary analysis, and stress responses of the GRAS gene family in castor beans. Int J Mol Sci. 2016;17:1004.

\section{Publisher's Note}

Springer Nature remains neutral with regard to jurisdictional claims in published maps and institutional affiliations.

Ready to submit your research? Choose BMC and benefit from:

- fast, convenient online submission

- thorough peer review by experienced researchers in your field

- rapid publication on acceptance

- support for research data, including large and complex data types

- gold Open Access which fosters wider collaboration and increased citations

- maximum visibility for your research: over $100 \mathrm{M}$ website views per year

At $\mathrm{BMC}$, research is always in progress.

Learn more biomedcentral.com/submissions 